\title{
Circuit Modeling and Performance Analysis of Multi-Walled Carbon Nanotube Interconnects
}

\author{
Hong Li, Student Member, IEEE, Wen-Yan Yin, Senior Member, IEEE, \\ Kaustav Banerjee, Senior Member, IEEE, and Jun-Fa Mao, Senior Member, IEEE
}

\begin{abstract}
Metallic carbon nanotubes (CNTs) have received much attention for their unique characteristics as a possible alternative to $\mathrm{Cu}$ interconnects in future ICs. Until this date, while almost all fabrication efforts have been directed toward multiwalled CNT (MWCNT) interconnects, there is a lack of MWCNT modeling work. This paper presents, for the first time, a detailed investigation of MWCNT-based interconnect performance. A compact equivalent circuit model of MWCNTs is presented for the first time, and the performance of MWCNT interconnects is evaluated and compared against traditional $\mathrm{Cu}$ interconnects, as well as Single-Walled CNT (SWCNT)-based interconnects, at different interconnect levels (local, intermediate, and global) for future technology nodes. It is shown that at the intermediate and global levels, MWCNT interconnects can achieve smaller signal delay than that of $\mathrm{Cu}$ interconnects, and the improvements become more significant with technology scaling and increasing wire lengths. At $1000-\mu \mathrm{m}$ global or $500-\mu \mathrm{m}$ intermediate level interconnects, the delay of MWCNT interconnects can reach as low as $15 \%$ of $\mathrm{Cu}$ interconnect delay. It is also shown that in order for SWCNT bundles to outperform MWCNT interconnects, dense and high metallic-fraction SWCNT bundles are necessary. On the other hand, since MWCNTs are easier to fabricate with less concern about the chirality and density control, they can be attractive for immediate use as horizontal wires in VLSI, including local, intermediate, and global level interconnects.
\end{abstract}

Index Terms-Carbon nanotube (CNT), circuit model, interconnect, multi-walled CNT (MWCNT), performance analysis, signal delay, single-walled CNT (SWCNT).

\section{INTRODUCTION}

C ARBON nanotubes (CNTs) have aroused a lot of interest in their applicability as VLSI interconnects of the future [1] because of their extremely desirable electrical and thermal

Manuscript received August 9, 2007; revised January 28, 2008. This work was supported in part by the National Science Fund for Creative Research Groups (60521002) via Shanghai Jiao Tong University, by the Grant of NSFC (90607011) of China, and by the National Institute of Standards and Technology, USA, under Grant 70NANB5H1215. The review of this paper was arranged by Editor M. Reed.

$\mathrm{H}$. Li is with the Center for Microwave and RF Technologies, School of Electronic, Information and Electrical Engineering, Shanghai Jiao Tong University, Shanghai 200240, China, and also with the Department of Electrical and Computer Engineering, University of California, Santa Barbara, CA 93106 USA (e-mail: hongli@ece.ucsb.edu).

W.-Y. Yin and J.-F. Mao are with the Center for Microwave and RF Technologies, School of Electronic, Information and Electrical Engineering, Shanghai Jiao Tong University, Shanghai 200240, China (e-mail: wyyin@sjtu.edu.cn; jfmao@sjtu.edu.cn).

K. Banerjee is with the Department of Electrical and Computer Engineering, University of California, Santa Barbara, CA 93106, USA (e-mail: kaustav@ ece.ucsb.edu)

Color versions of one or more of the figures in this paper are available online at http://ieeexplore.iee.org.

Digital Object Identifier 10.1109/TED.2008.922855

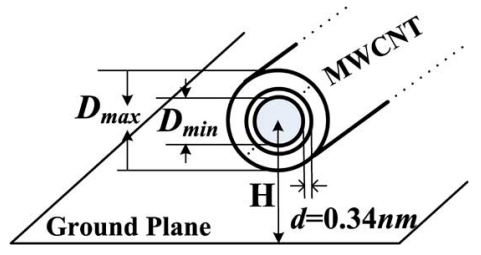

Fig. 1. Geometry of an MWCNT on a ground plane.

properties [2], [3]. CNTs have long mean free paths (MFPs) on the order of several micrometers (as compared to $40 \mathrm{~nm}$ for $\mathrm{Cu}$ at room temperature), which provide low resistivity and possible ballistic transport in short-length interconnects [4]. More importantly, an isolated CNT can carry current densities in excess of $10^{10} \mathrm{~A} / \mathrm{cm}^{2}$ without any signs of damage even at an elevated temperature of $250{ }^{\circ} \mathrm{C}$ [5], thereby eliminating electromigration reliability concerns that plague nanoscale $\mathrm{Cu}$ interconnects [6]-[8].

CNTs are sheets of graphene rolled up as hollow cylinders. CNTs can be classified as single-walled (SWCNTs, with only one shell and diameter ranging from 0.4 to $4 \mathrm{~nm}$ ) and multiwalled (MWCNTs, with several concentric shells and diameter ranging from several nanometers to tens of nanometers). While SWCNTs can be either metallic or semiconducting depending on their chirality (the direction in which they get rolled up), giving rise to zigzag (mostly semiconducting), armchair (metallic), or chiral nanotubes (mostly semiconducting), MWCNTs are always metallic. Moreover, MWCNTs have similar current carrying capacity (as metallic SWCNTs) but are easier to fabricate than SWCNTs due to easier control of the growth process. However, due to its simple structure, SWCNTs can be modeled more easily than MWCNTs. In fact, while most CNT interconnect fabrication efforts have targeted MWCNTs [9]-[12], nearly all modeling efforts are focused on the analysis of isolated SWCNTs [13] or SWCNT bundles [14]-[17]. These studies indicate that compared to $\mathrm{Cu}$ wires, SWCNT bundles can provide significant improvement in delay for long (global and intermediate levels) interconnects.

However, few research efforts have addressed the modeling and performance analysis of MWCNT-based interconnects due to their complexity in both structure and characterization. MWCNTs consist of several coaxial CNT shells (as shown in Fig. 1) and each shell in an MWCNT can have different chiralities depending on the direction they are rolled up, which implies that the shells in MWCNT may be metallic or semiconducting.

It is important to note that, until recently, MWCNTs had not been reported to exhibit conductance values comparable to 


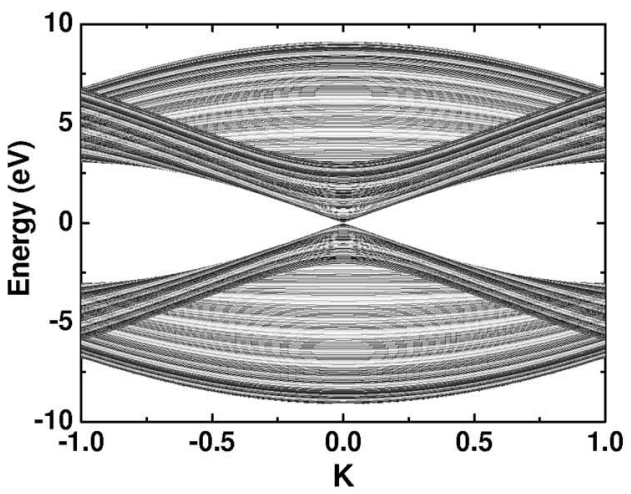

(a)

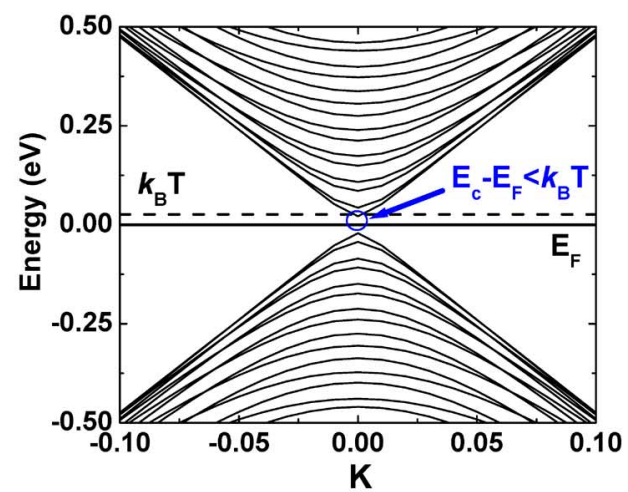

(b)

Fig. 2. (a) Band structure of a zigzag $(256,0)$ carbon nanotube, and (b) the band structure after zoom in for clarity. $K$ is the normalized wave vector. Each line denotes a subband of CNT shell. In the circular area (around $K=0$ ), the energy difference between the conduction band edge $\left(E_{c}\right)$ and Fermi-level $E_{F}$ is smaller than $k_{B} T$.

SWCNTs and, therefore, were considered less desirable than SWCNTs for interconnect applications. This can be attributed to the fact that in the early experiments, only the outermost shell in MWCNTs was contacted with metal, whereas the inner shells were isolated from the contact, and therefore had little effect on the conductance [18]. However, more recently, it has been found that multiple shells in an MWCNT can contribute to conductance if proper end contacts can be made. In [11], [12], and [19], properly contacted MWCNTs have been implemented to achieve low resistance with the contribution of inner shells. Moreover, low resistances achieved in these works also indicate that the contact resistance between metal and the nanotube can be relatively small.

In order to analyze the performance of MWCNT interconnects, their equivalent distributed circuit model needs to be derived. In [20], a compact model of conductivity of MWCNTs is proposed, which is applicable for various diameters and lengths. However, it is important to note that the performance of interconnects is usually affected by other interconnect parasitic parameters, such as capacitance and inductance. Furthermore, as it will be shown in Section II, the circuit model of MWCNTs is much more complex than the traditional $R L C$ distributed interconnect model. Not only does each shell in MWCNTs have different parameters but also there are couplings between neighboring shells. Hence, simply adding up the conductance of each shell is insufficient for predicting the overall performance of MWCNTs. Coupling between neighboring shells has been considered in [21], where the crosstalk effect of MWCNT was discussed. However, they did not consider the diameter dependence of conducting channels and assumed all shells in an MWCNT to have identical number of conducting channels as in an SWCNT $(=2)$, which is not correct, particularly for large-diameter MWCNTs.

In this paper, an equivalent distributed circuit model of MWCNTs is proposed, in which the diameter dependence of MFP and conducting channel are incorporated. Intershell coupling and tunneling effects are also taken into account. Based on the interconnect geometry predicted by ITRS 2005 [22], the proposed model is implemented and employed for comprehensive performance comparisons between MWCNT interconnects, $\mathrm{Cu}$ wires, and SWCNT bundles at the local, intermediate, and global interconnect levels. The results can provide guidelines for the development of CNT-based interconnect technology in future IC applications.

This paper is organized as follows. Section II introduces the equivalent circuit model for an MWCNT, which can serve as the basis for the performance prediction of MWCNT interconnects. In Section III, resistivity and performance comparison between MWCNT interconnects and $\mathrm{Cu}$ interconnects are carried out based on a typical interconnect structure. Further results of performance comparison between MWCNT and SWCNT bundles are presented in Section IV. Finally, conclusions are drawn in Section V.

\section{EQuivalent Distributed Circuit MOdel of MWCNT}

An isolated MWCNT on an infinite ground plane is shown in Fig. 1. The separation between the nanotube center and the ground is $H$, the diameter of the outermost shell is $D_{\text {max }}$, the innermost shell's diameter is $D_{\text {min }}$, and the interval between two adjacent shells is $d \approx 0.34 \mathrm{~nm}$, which is the van der Waals gap.

\section{A. Conductive Property of MWCNT}

Because of its large diameter, the shells of an MWCNT would be conductive even if they are of semiconducting chirality. This can be explained from the band structure of a large-diameter nanotube. Fig. 2(a) shows the band structure of a zigzag CNT shell, whose diameter is $20 \mathrm{~nm}$ and can be characterized by the chiral index $(256,0)$. It is a semiconducting structure and has an energy gap, as shown in Fig. 2(b). At room temperature $(T=300 \mathrm{~K})$, the thermal energy, denoted by $k_{B} T$, is equal to $0.0258 \mathrm{eV}$ approximately, where $k_{B}$ is the Boltzmann constant. From Fig. 2(b), it can be observed that for $(256,0)$ zigzag CNT, the energy difference between the conduction band edge $\left(E_{C}\right)$ and the Fermi level $E_{F}$ is smaller than $0.0258 \mathrm{eV}$, which indicates that when the diameter of CNT is around $20 \mathrm{~nm}$ or larger, these energy differences will be smeared by the environment temperature.

In addition, even if the energy difference between the subbands and $E_{F}$ is larger than $k_{B} T$, this energy difference is 
relatively small due to the large density of states in largediameter shells. The electrons in those subbands have reasonable probability $\left(f_{i}\right)$ to appear at $E_{F}$ following the Fermi-Dirac distribution function:

$$
f_{i}=\frac{1}{\exp \left(\left|E_{i}-E_{F}\right| / k_{B} T\right)+1}
$$

where $E_{i}$ is the highest (or lowest) value for the subbands below (or above) the Fermi level $E_{F}$. Since there are large number of subbands in large-diameter shells, their cumulative effect on the probability could be large, which implies that they could have large number of conducting channels. Thus, since MWCNTs have many large-diameter shells, they could turn out to be good electrical conductors and would be very attractive as possible interconnect materials.

\section{B. Number of Channels}

Based on (1), the number of conducting channels (spin degeneracy is already considered) in any shell can be obtained by adding up all the probabilities

$$
N_{\text {shell }}=\sum_{\text {subbands }} \frac{1}{\exp \left(\left|E_{i}-E_{F}\right| / k_{B} T\right)+1} .
$$

In further analysis, it is more convenient to use the approximated form of (2) given in [20] as

$$
N_{\text {shell }}(D) \approx a \cdot D+b, \quad D>3 \mathrm{~nm}
$$

where $D$ is the diameter of the shell, $a=0.0612 \mathrm{~nm}^{-1}$, and $b=0.425$. The error introduced by (3), due to different chiralities, is within $15 \%$ for all values of $D$.

In this paper, similar to the experimental measurements in [19] and modeling in [20], the ratio of $D_{\min } / D_{\max }$ can be assumed to be $1 / 2$, approximately. Thus, the number of shells $p$ of the MWCNT is determined by

$$
p=1+\operatorname{Inter}\left[\frac{\left(D_{\max }-D_{\max } / 2\right)}{2 d}\right]
$$

where "Inter[.]" indicates that only the integer part is taken into account. If we denote the shells from the outer to the inner as $1,2, \ldots, i, \ldots$, and $p$, the diameter of the $i^{\text {th }}$ shell is given by

$$
D_{i}=D_{\max }-2 d \cdot(i-1), \quad 1 \leq i \leq p
$$

where $d$ is the van der Waals gap. The innermost diameter in Fig. 1 is $D_{\min }=D_{\max }-2 d \cdot(p-1)$. Note that, although the ratio of $D_{\min } / D_{\max }$ is assumed to be $1 / 2, D_{\min }$ may be larger than $D_{\max } / 2$ because $D_{\max }$ may not be an integer multiple of $d$. The number of conducting channels of the $i^{\text {th }}$ shell is given by

$$
N_{i}=a \cdot D_{i}+b
$$

Hence, the total number of conducting channels is given by the sum of the conducting channels $\left(N_{i}\right)$ of all the shells. It is important to note that (3) and (6) are only applicable under

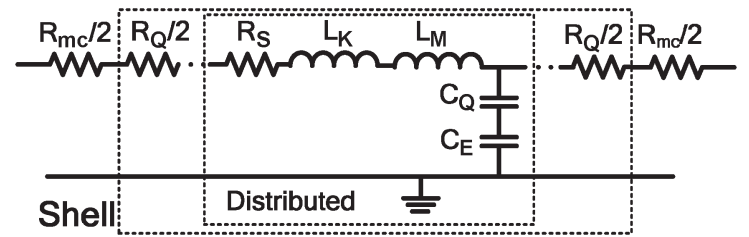

Fig. 3. Equivalent distributed circuit model of an individual shell. $R_{\mathrm{mc}}$ is the imperfect contact resistance, $R_{Q}$ is the quantum contact resistance, $R_{S}$ is the scattering-induced resistance, $L_{K}$ and $L_{M}$ are the kinetic and magnetic inductances, respectively, and $C_{Q}$ and $C_{E}$ are the quantum and electrostatic capacitances, respectively.

small voltage bias, which is known to be valid for interconnect analysis [14]-[17].

\section{C. $R, L$, and $C$ for an Individual Shell}

Having obtained the number of conducting channels for a given shell, one can obtain its circuit model by following a similar procedure used in the derivation of the model of SWCNTs, which is shown in Fig. 3.

1) Resistance: The resistance of a shell consists of three parts [4]: quantum contact resistance $R_{Q}$, scattering-induced resistance $R_{S}$, and imperfect contact resistance $R_{\mathrm{mc}}$. Note that $R_{S}$ only occurs if the length of the nanotube (shell) is larger than the electron MFP. $R_{Q}$ and $R_{S}$ are intrinsic, and $R_{\mathrm{mc}}$ is due to fabrication process. The value of the intrinsic conductance $(G)$ is determined by

$$
G^{-1}=R_{Q}+R_{S} \cdot L=\frac{h}{2 e^{2} N}+\frac{h}{2 e^{2} N} \cdot \frac{L}{\lambda}
$$

where $h / 2 e^{2} \sim 12.9 \mathrm{k} \Omega$, and $L, \lambda$, and $N$ are the length, MFP, and number of conducting channels of the shell, respectively. The imperfect contact resistance $R_{\mathrm{mc}}$ can range from zero to hundreds of kilo-Ohms for different growth processes. Recently, as demonstrated in [11], [12], and [19], $R_{\mathrm{mc}}$ in MWCNT could be very small compared to the total resistance.

From (7), it can be observed that the value of MFP plays an important role in determining the resistance of the nanotube. It has been proven that the MFP of nanotube is proportional to the diameter, both for metallic and semiconducting nanotubes.

For metallic nanotube, it is derived in [23] that

$$
\lambda=D \cdot \sqrt{3} \pi \psi^{2} /\left(2 \sigma_{\varepsilon}^{2}+9 \sigma_{\psi}^{2}\right)
$$

where $\psi$ is the nearest neighbor tight-binding parameter, $\varepsilon$ is the on-site energies, and $\sigma_{\varepsilon}$ and $\sigma_{\psi}$ are the variances of $\varepsilon$ and $\psi$, respectively. $\sigma_{\varepsilon}, \sigma_{\psi}$, and $\psi$ can be considered to be constant.

For semiconducting nanotube, we can obtain this linear relationship from [24], as given by

$$
\lambda=D \cdot v_{F} /(\alpha T)
$$

where $v_{F}$ is the Fermi velocity of CNTs $\left(\sim 8 \times 10^{5} \mathrm{~m} / \mathrm{s}\right), \alpha$ is the coefficient of scattering rate, and $T$ is the temperature.

Thus, irrespective of the nature of shells in an MWCNT, metallic or semiconducting, its MFP always depends on its 
diameter. For a typical SWCNT with $D=1 \mathrm{~nm}$, the MFP is about $1 \mu \mathrm{m}$ [17], [20]. ${ }^{1}$ Thus, we can have $\lambda \approx 1000 D$.

2) Inductance: For a CNT, there are magnetic inductance and kinetic inductance [13] (as shown in Fig. 3). The magnetic and kinetic inductances per unit length of a shell with configuration shown in Fig. 1 are

$$
\begin{aligned}
L_{\text {magnetic }} & =\frac{\mu}{2 \pi} \cosh ^{-1}(2 H / D) \\
L_{K / \text { channel }} & =\frac{h}{2 e^{2} v_{F}} \times \frac{1}{2} \approx 8 \mathrm{nH} / \mu \mathrm{m} \\
L_{K / \text { shell }} & =L_{K / \text { channel }} /(a \cdot D+b) .
\end{aligned}
$$

For $3 \mathrm{~nm}<D<100 \mathrm{~nm}$, the total kinetic inductance $\left(L_{K / \text { shell }}\right)$ may decrease from 13 to $1 \mathrm{nH} / \mu \mathrm{m}$. Meanwhile, the magnetic inductance of each shell described in (10) is a weak function of the factor $H / D$. For $1<H / D<100$, the magnetic inductance ranges from 0.2 to $1.2 \mathrm{pH} / \mu \mathrm{m}$, which is much smaller than the kinetic inductance. Hence, the magnetic inductance has been ignored in this paper.

3) Capacitance: The capacitance of a CNT also has two parts: quantum capacitance $C_{Q}$ and electrostatic capacitance $C_{E}$ (as shown in Fig. 3). According to the result in [13], quantum capacitance per unit length of a shell can be derived as

$$
\begin{aligned}
C_{Q / \text { channel }} & =2 \times \frac{2 e^{2}}{h v_{F}} \approx 193 \mathrm{aF} / \mu \mathrm{m} \\
C_{Q / \text { shell }} & =C_{Q / \text { channel }} \times(a \cdot D+b) .
\end{aligned}
$$

The electrostatic capacitance $\left(C_{E}\right)$ depends on the geometry of the structure and will be discussed in the next section.

\section{MWCNT Model}

An MWCNT consists of many concentric shells, which can be viewed as several shells in parallel. It may seem similar to an SWCNT bundle consisting of many nanotubes in parallel, but in fact, it has significant differences with an SWCNT bundle. For an SWCNT bundle, since all nanotubes in the bundle are assumed to have identical diameter, they can be easily transformed into a simple circuit model [14]-[17]: The effective resistance and kinetic inductance per tube are divided by the number of SWCNTs, and the quantum capacitance per tube is multiplied by the number of SWCNTs. However, in an MWCNT, different shells have different diameters, which translate to different channel numbers and MFPs, resulting in different circuit parameters. Hence, the parameters of each shell cannot be combined in a simple way as in the case of SWCNT bundles.

Moreover, since circuit parameters of different shells vary in MWCNTs, the potentials of different shells cannot be assumed to be equal as in the case of SWCNT bundles, which induces shell-to-shell capacitive coupling. This coupling capacitance is akin to electrostatic capacitance and will be very large due to the small separation between two adjacent shells. The shell-to-

\footnotetext{
${ }^{1}$ The value of MFP reported in these papers is based on measurements and is smaller than theoretical predictions.
}

shell capacitance per unit length $\left(C_{S}\right)$ can be obtained by using the coaxial capacitance formula [25]

$$
C_{S}=\frac{2 \pi \varepsilon}{\ln \left(D_{\text {out }} / D_{\text {in }}\right)}=\frac{2 \pi \varepsilon}{\ln \left[D_{\text {out }} /\left(D_{\text {out }}-2 d\right)\right]}
$$

where $D_{\text {out }}$ and $D_{\text {in }}$ are the diameters of the outer and inner shells of adjacent coaxial shells, and $d$ equals $0.34 \mathrm{~nm}$.

One important physical effect in MWCNTs is the tunneling effect between two adjacent shells. This effect still needs more investigation since there are some conflicting conclusions in the literature. In [26], it is claimed that the interaction between shells in an idealized MWCNT can be assumed to be neglegible. Theoretical analysis in [27] also shows that the tunneling current between shells of a defect-free infinitely long MWCNT is vanishingly small in general, whereas measurements in $[28]^{2}$ show that the conductance between two shells is about $\sim(10 \mathrm{k} \Omega)^{-1} / \mu \mathrm{m}$, which yields a radial resistivity value of $\sim 1 \Omega \cdot \mathrm{m}$. Note that this measured resistivity is only for the shell interval of $d=0.34 \mathrm{~nm}$. However, the tunneling effect would be exponentially dependent on the shell interval. Since the shell interval of MWCNT can be assumed to be $0.34 \mathrm{~nm}$, here, we introduce a normalized tunneling conductivity $(\sigma)$ as a parameter that includes shell interval dependence. The value of this normalized tunneling conductivity then can be calculated as $0.3\left(\mu \Omega \cdot \mathrm{cm}^{2}\right)^{-1}$ based on measurements [28]. The intershell tunneling conductance per unit length can be derived as

$$
G_{T}=\sigma \cdot \pi D
$$

where $\sigma$ and $D$ are normalized tunneling conductivity at $d=0.34 \mathrm{~nm}$ and the shell diameter, respectively. In our model, in order to analyze the impact of tunneling effect on the performance, we employed a range of the normalized tunneling conductivity from no tunneling effect $(\sigma=0)$ to the measurement value of $0.3\left(\mu \Omega \cdot \mathrm{cm}^{2}\right)^{-1}$. Note that the tunneling conductance is proportional to the diameter. This is because there are more atoms in a larger diameter shell and tunneling is more likely to take place.

Another effect is the magnetic coupling between the shells in an MWCNT. Because of the large diameter and small thickness, each shell can be treated as an ideal sheet cylinder with zero thickness. The mutual inductance per unit length between different shells can be derived from the equations in [29] as

$$
M_{\text {shell }}=\frac{\mu}{2 \pi}\left(\ln \frac{4 L}{D_{\text {out }}}-1+\frac{D_{\text {out }}+D_{\text {in }}}{\pi L}\right)
$$

where $L$ is the length, and $D_{\text {out }}$ and $D_{\text {in }}$ are the diameters of the outer and inner shells of the coaxial structure, respectively. From (16), the mutual inductance between shells can be estimated to be around $2 \mathrm{pH} / \mu \mathrm{m}$, which is much smaller than the kinetic inductance of each shell (of the order of nanohenry per micrometer). Hence, the mutual inductance is ignored in our analysis.

Based on the aforementioned analysis, an equivalent distributed circuit model for MWCNT interconnect is proposed, as

\footnotetext{
${ }^{2}$ Measurements automatically take defects into account.
} 


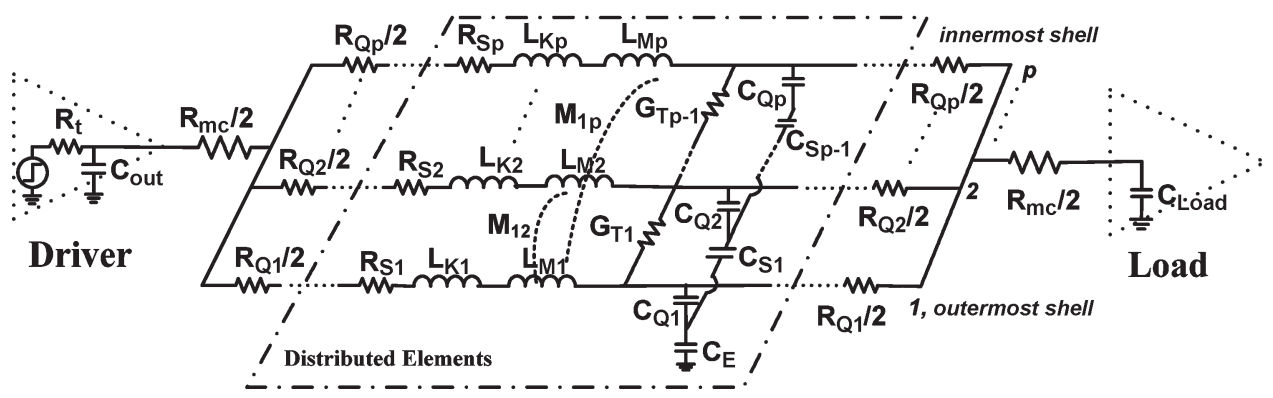

Fig. 4. Equivalent distributed circuit model of an MWCNT with $p$ shells. Aside from the lumped imperfect contact resistance $R_{\mathrm{mc}}$ and lumped quantum resistance $R_{Q}$ per shell, each shell has distributed scattering resistance $R_{S}$, kinetic inductance $L_{K}$, magnetic inductance $L_{M}$, mutual inductance $M$, and quantum capacitance $C_{Q}$. Only the outermost shell has electrostatic capacitance $C_{E}$ with the ground. The shell-to-shell capacitance $C_{S}$ and the tunneling conductance $G_{T}$ only have $p-1$ distributed components for the entire MWCNT. $R_{t}$ and $C_{\text {out }}$ are the effective resistance and output capacitance of the driver, respectively, and $C_{\text {load }}$ is the input capacitance of the load.

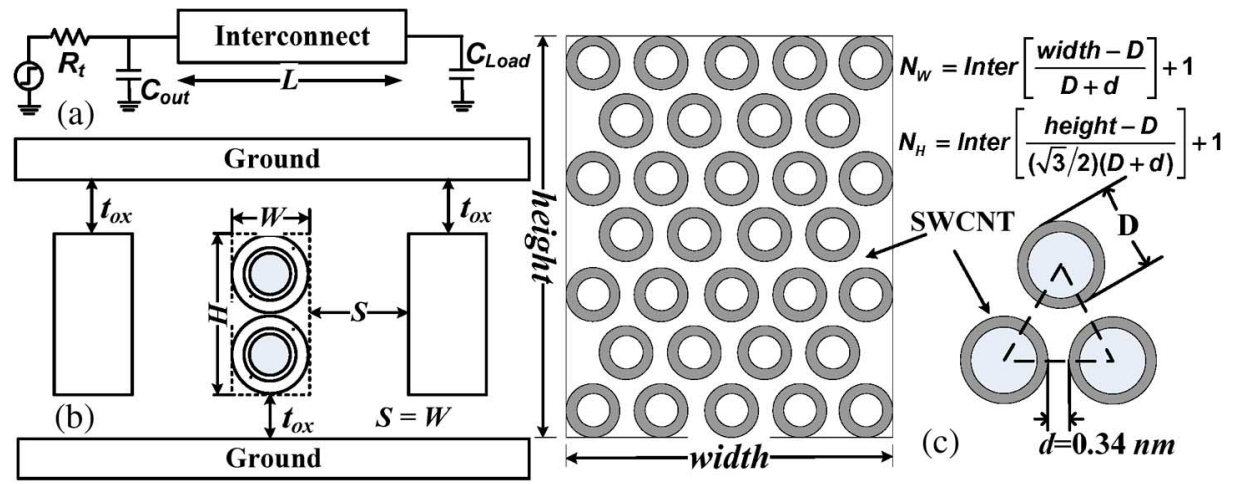

Fig. 5. (a) Schematic of circuit used for performance evaluation. The "interconnect" can be replaced by an equivalent circuit model for MWCNTs, Cu wires, or SWCNT bundles. (b) Cross section of a typical interconnect configuration in advanced IC designs. In this figure, the aspect ratio is two. (c) Cross-sectional view of densely packed SWCNT bundles. $N_{W}$ and $N_{H}$ are the number of CNTs along the width and height, respectively.

shown in Fig. 4. The quantum resistance $R_{Q}$ and scattering resistance $R_{S}$ are obtained by using (7). Kinetic inductance $L_{K}$, quantum capacitance $C_{Q}$, shell-to-shell capacitance $C_{S}$, and tunneling conductance $G_{T}$ are given by (12)-(15), respectively. The quantum capacitance $C_{Q}$ is in series with electrostatic capacitance (including shell-to-shell capacitance $C_{S}$ and ground capacitance $C_{E}$ ) [13]. Note that there is only one total ground capacitance $C_{E}$ in the model shown in Fig. 4. This is because only the outermost shell has the electrostatic interaction with the ground, whereas the inner shells are shielded. For shellto-shell capacitance and tunneling, only neighboring shells are involved. Therefore, for $p$ shells, there is only $p-1$ number of $C_{S}$ and $G_{T}$. The equivalent model in Fig. 4 can be employed for circuit simulation using SPICE.

\section{MWCNT Versus Cu INTERCONNECTS}

In this section, the delay of an MWCNT interconnect is estimated and compared with the traditional $\mathrm{Cu}$ interconnect. We consider a typical interconnect structure shown in Fig. 5(a) and (b), where $R_{t}$ and $C_{\text {out }}$ are the equivalent output resistance and capacitance of the gate driver, respectively, and $C_{\text {load }}$ is the input capacitance of the load gate. The input excitation is assumed to be a step signal. All interconnect parameters used in simulations are obtained from ITRS 2005 [22], as summarized in Table I. In this paper, the diameter of MWCNT is set equal to the minimum width of interconnect (as shown in Table I) at each technology node, unless specified otherwise.
TABLE I

ITRS 2005 BASED SIMULATION PARAMETERS

\begin{tabular}{|c|c|c|c|c|}
\hline Technolc & y Node $(\mathrm{nm})$ & 32 & 22 & 14 \\
\hline \multirow{6}{*}{$\begin{array}{c}\text { Local \& } \\
\text { Intermediate }\end{array}$} & Width $W(n m)$ & 32 & 22 & 14 \\
\hline & $A / R *$ & 2 & 2 & 2 \\
\hline & ILD Thickness $t_{o x}(n m)$ & 54.4 & 39.6 & 25.2 \\
\hline & $\rho_{C u}(u \Omega \cdot c m)$ & 4.83 & 6.01 & 8.19 \\
\hline & $C_{C u}(p F / m)^{* *}$ & 144.93 & 131.01 & 111.83 \\
\hline & $C_{M W C N T}(p F / m) * *$ & 130.15 & 117.70 & 100.51 \\
\hline \multirow{6}{*}{ Global } & Width $W(\mathrm{~nm})$ & 48 & 32 & 21 \\
\hline & $A / R *$ & 3 & 3 & 3 \\
\hline & ILD Thickness $t_{o x}(\mathrm{~nm})$ & 110.4 & 76.8 & 52.5 \\
\hline & $\rho_{C u}(\mu \Omega \cdot c m)$ & 3.52 & 4.2 & 5.38 \\
\hline & $C_{C u}(p F / m)^{* *}$ & 179.78 & 163.3 & 139.03 \\
\hline & $C_{M W C N T}(p F / m)^{* *}$ & 163.81 & 148.9 & 126.78 \\
\hline \multirow{3}{*}{$\begin{array}{l}\text { Minimum } \\
\text { Sized Gate }\end{array}$} & $R_{t}(k \Omega)$ & 13.85 & 16.67 & 18.33 \\
\hline & $C_{\text {out }}(f F)$ & 0.07 & 0.049 & 0.03 \\
\hline & $C_{\text {in }}(f F)$ & 0.25 & 0.14 & 0.065 \\
\hline \multicolumn{2}{|l|}{$K_{I L D}$} & 2.25 & 2.05 & 1.75 \\
\hline \multicolumn{5}{|c|}{$\begin{array}{l}\text { The aspect ratio }(A / R) \text { for local (intermediate) and global level interconnec } \\
\text { in ITRS are in the range of } 1.9-2.0 \text { and } 2.5-2.8 \text {. For convenience, we set } \\
\text { them as } 2 \text { and } 3 \text {, respectively. }\end{array}$} \\
\hline
\end{tabular}

The capacitance of a $\mathrm{Cu}$ wire and MWCNT bundle is calculated by using the modified FEM method [30], and the inductance of $\mathrm{Cu}$ interconnect is calculated using FastHenry [31]. In the simulations, $\mathrm{Cu}$ interconnects are simulated using the rigorous distributed "W" model of HSPICE [32], whereas the MWCNTs are simulated using the equivalent circuit shown 


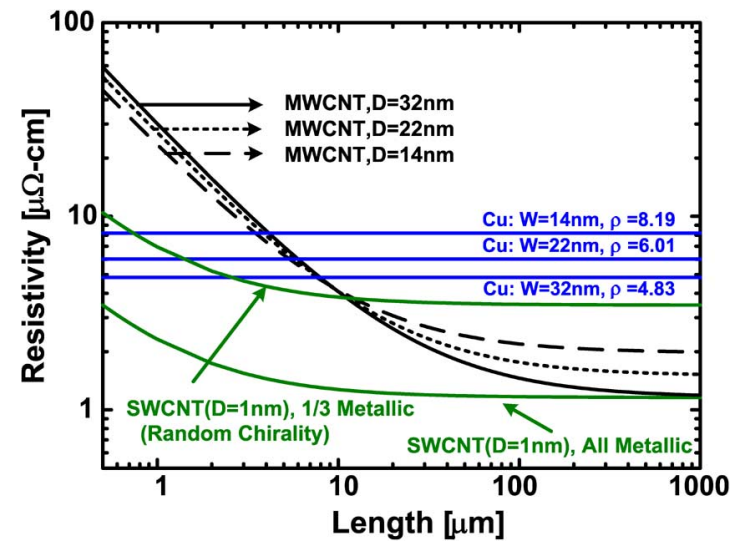

Fig. 6. Comparison of resistivity among MWCNTs with various diameters, $\mathrm{Cu}$ wires with different dimensions, and SWCNT bundles with different chiralities. Dimensions of $\mathrm{Cu}$ wires are adopted from ITRS as in Table I. SWCNT bundles are assumed to be densely packed.

in Fig. 4, with the distributed elements repeated over 200300 subsections in order to get comparable accuracy.

\section{A. Comparison of Equivalent Resistivity}

In order to comprehend the intrinsic differences between MWCNT, SWCNT, and $\mathrm{Cu}$ interconnects, their equivalent resistivities are compared. This is helpful for understanding the subsequent results of performance comparisons between MWCNTs, $\mathrm{Cu}$, and SWCNTs at different interconnect levels.

Based on the model and equation (7) described in Section II, the conductance of MWCNTs can be obtained by summing up the conductances of all shells. Similar to the study in [20], we employed an equivalent resistivity of MWCNTs and compared it with that of $\mathrm{Cu}$. The diameters of MWCNTs are chosen according to typical interconnect widths, as shown in Table I. The comparison result is shown in Fig. 6. For $\mathrm{Cu}$ wires, the resistivities of local and intermediate level wires are adopted from ITRS as in Table I. For SWCNT bundles, we considered them to be densely packed but for two cases: 1) All the CNTs in the bundle are metallic, and 2) CNTs in the bundle have random charities, which implies that only $1 / 3$ of CNTs are metallic and contribute to conductance.

It can be observed that for long lengths $(>10 \mu \mathrm{m})$, the resisitivity of MWCNTs could be several times lower than that of $\mathrm{Cu}$ wire and becomes increasingly comparable to that of SWCNT bundles.

\section{B. Global Interconnects}

Lengths of global interconnects can be on the order of millimeters. Repeaters are normally inserted in order to increase the drive capability and reduce the signal delay. The size of repeaters is often much larger than the minimum sized gates [33]. In this paper, we consider the interconnect section between two such adjacent repeaters [as shown in Fig. 5(a)], whose size is set to be 100 times the minimum size. It is important to note that if different repeater sizes are used, (such as the optimal size rather than the fixed value used here), the absolute values of the following comparisons would be different, however, the trends of the comparative results will remain the same.
The comparison of delay between MWCNT and $\mathrm{Cu}$ interconnects at the global level is shown in Fig. 7(a). It can be observed that the delay $(\tau)$ of MWCNT interconnects is smaller than that of $\mathrm{Cu}$ interconnects and that the improvement in delay performance in the case of MWCNT interconnects increases for longer lengths. At $1000 \mu \mathrm{m}$, the delay of MWCNT interconnect is only around $15 \%$ of that of $\mathrm{Cu}$ interconnect. This indicates that MWCNT can improve the circuit performance significantly in future interconnect applications. It is also shown that the normalized tunneling conductivity value of $0.3\left(\mu \Omega \cdot \mathrm{cm}^{2}\right)^{-1}$ has minor impact on the performance enhancement. It is instructive to note that for MWCNT global wires, performance estimation based solely on the resistivity values (Fig. 6) or on a simple $R C$ (or simple $R L C$ ) delay model will induce large errors since the equivalent circuit model for MWCNTs is more complex, as shown in Fig. 4.

In the aforementioned analysis, the width of global interconnects is assumed to be the minimum width predicted by ITRS (shown in Table I). In practice, the width of global interconnects is normally much wider than the minimum value $\left(3 \sim 10 \times W_{\min }\right)$ in order to reduce the resistance of the wire and thus decrease delay, IR drop and power consumption [34], [35]. Fig. 7(b) shows the signal delay ratios of an MWCNT interconnect to a $\mathrm{Cu}$ interconnect at the global level at the 22-nm technology node for three different widths $\left(W_{\min }, 3 \cdot W_{\min }\right.$, and $\left.6 \cdot W_{\min }\right)$. The outer diameter of MWCNT is set to be the minimum value $(32 \mathrm{~nm})$. Thus, the number of MWCNTs in these three scenarios are 3,9 , and 18 , respectively. It can be observed that the delay ratio increases with increasing wire width (i.e., the performance enhancement of MWCNT interconnect decreases).

However, if the width of global interconnect is increased, the diameter of MWCNTs could also be increased accordingly. The impact of increasing the MWCNT diameter is shown in Fig. 7(c). In this figure, the wire width is kept constant $\left(6 \cdot W_{\min }\right)$, whereas MWCNTs of three diameters $(24,32$, and $48 \mathrm{~nm}$ ) are considered. The numbers of MWCNTs in these scenarios are 32,18 , and 8 , respectively. Although larger diameter decreases the number of MWCNTs contained in the interconnect cross section, it effectively enhances the circuit performance particularly for longer wires. This is due to the fact that for larger diameters, the resistivity of MWCNT for long lengths (as shown in Fig. 6) becomes smaller, which counteracts the negative effect of decreasing number of MWCNTs.

\section{Intermediate Interconnects}

Although the lengths of intermediate level interconnects are often shorter than that of global interconnects, repeaters are still required for intermediate level interconnects. In this paper, the size of repeater is chosen to be 50 times the minimum size for intermediate interconnects (length ranges from 20 to $500 \mu \mathrm{m}$ ).

Fig. 8(a) shows the delay comparison of intermediate MWCNT and $\mathrm{Cu}$ interconnects for different technology nodes. It is found that similar to global level interconnects, the performance of MWCNT interconnects is much better than that of $\mathrm{Cu}$, and the improvement in delay increases with increasing length and technology scaling. At 500- $\mu \mathrm{m}$ length, the delay 


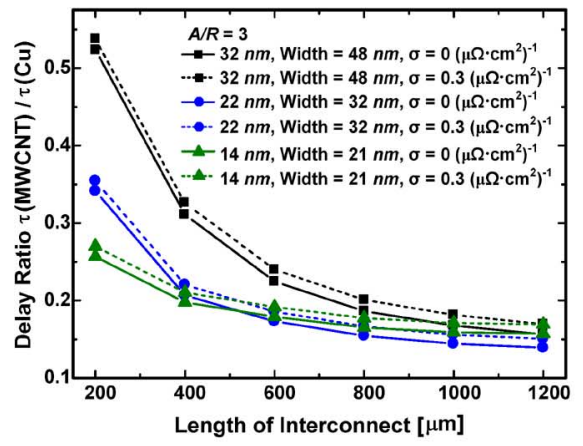

(a)

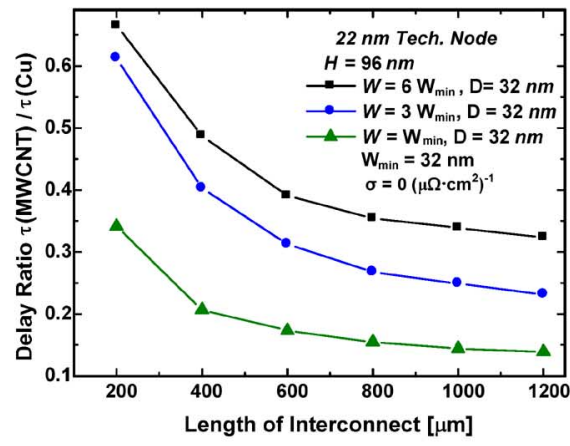

(b)

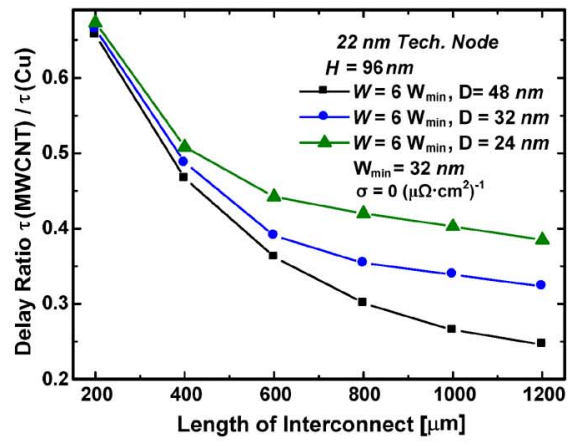

(c)

Fig. 7. Signal delay ratios of MWCNT interconnect with respect to $\mathrm{Cu}$ interconnect at the global level for (a) minimum interconnect widths as in Table I, (b) different widths but the same MWCNT diameter, and (c) identical widths but different MWCNT diameters. In (a), the normalized tunneling conductivity ( $\sigma$ ) value between adjacent shells is assumed to be 0 and $0.3\left(\mu \Omega \cdot \mathrm{cm}^{2}\right)^{-1}$, represented by solid and broken lines, respectively.

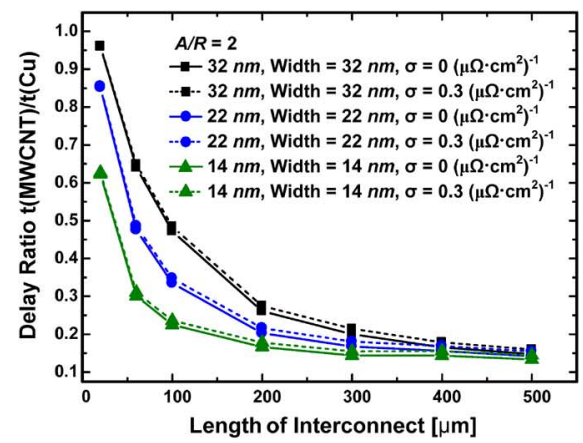

(a)

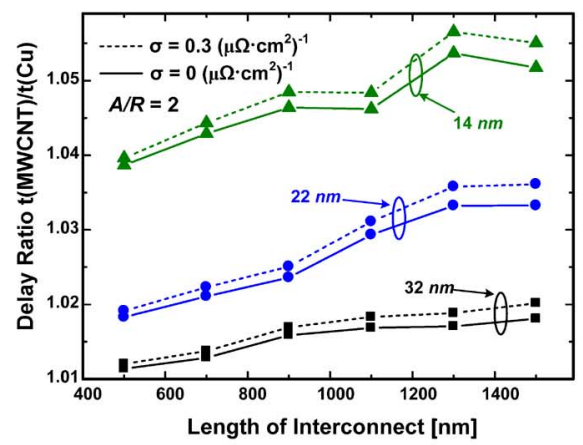

(b)

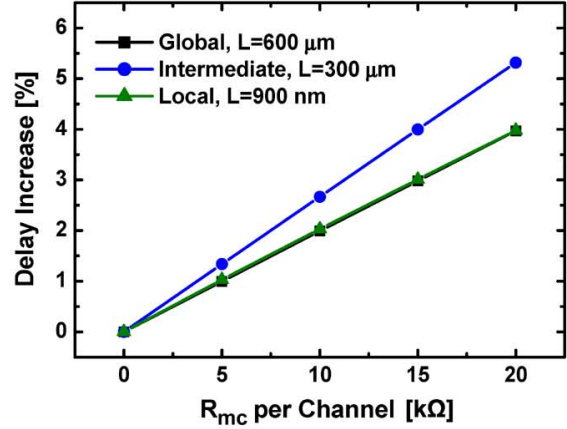

(c)

Fig. 8. Signal delay ratios of MWCNT interconnect with respect to Cu interconnect at the (a) intermediate level, and (b) local level for 32-, 22-, and 14-nm technology nodes. The normalized tunneling conductivity $(\sigma)$ value between adjacent shells is assumed to be 0 and $0.3\left(\mu \Omega \cdot \mathrm{cm}^{2}\right)^{-1}$, represented by solid and broken lines, respectively. (c) The impact of $R_{\mathrm{mc}}$ per conducting channel on the delay increase in local, intermediate, and global level MWCNT interconnects.

of MWCNT interconnect can reach as low as $15 \%$ of that of $\mathrm{Cu}$ wire. Similar to global cases, the tunneling conductance of MWCNT at intermediate level shows little impact on the performance enhancement.

The significantly smaller signal delays of MWCNT interconnects at the global and intermediate levels have significant implications for nanoscale VLSI. Smaller signal delay can provide higher operating frequency and faster circuit speed. From repeater insertion perspective, interconnects with smaller delay can endure longer distances between adjacent repeaters and therefore need fewer repeaters, which can further save chip area and power consumption. Hence, using MWCNT interconnects (in place of $\mathrm{Cu}$ ) can achieve higher performance and lower power in integrated circuits.

\section{Local Interconnects}

Local interconnects have the smallest cross section and largest resistance per unit length, as compared to either intermediate or global interconnects. They are often used for connecting nearby gates or devices, with lengths on the order of micrometers. The drivers at the local level are usually minimum sized gates and have fanout $>1$. A typical fanout scenario is FO4 (fanout of four), which is considered in this paper.

For the smallest MWCNT $\left(D_{\max }=14 \mathrm{~nm}\right)$ concerned, the smallest MFP associated with the innermost shell $\left(D_{\min }=\right.$ $7 \mathrm{~nm}$ ) is about $7 \mu \mathrm{m}$ [known from (8) or (9)], which is much larger than the typical length of local interconnects. Under such a condition, it can be assumed that the MWCNT is in ballistic transport regime with no scattering effects. Hence, the scattering-induced resistance $R_{S}$ can be ignored for local level interconnects. Fig. 8(b) shows the comparison of signal delay between local level MWCNT and $\mathrm{Cu}$ interconnects. Unlike the case of intermediate or global level, the delay of MWCNT interconnects at the local level is marginally larger than that of $\mathrm{Cu}$ (by $\sim 1 \%-6 \%$ ). From Fig. 6, one can observe that the resistivity of MWCNTs is much larger than that of $\mathrm{Cu}$ wires for lengths around $1 \mu \mathrm{m}$. However, due to small driver size, and thereby large driver resistance, the shortcoming of local level MWCNT interconnects is overshadowed by the driver gates. This result also indicates that only resistivity comparison, as presented in [20], is insufficient for the accurate analysis of circuit performance.

\section{E. Impact of $R_{\mathrm{mc}}$}

In previous analysis (Section III-B-D), the imperfect contact resistance $\left(R_{\mathrm{mc}}\right)$ of MWCNT interconnect was considered to be zero. Although it has been pointed out in Section II-C that this imperfect contact resistance could be very small, it is analyzed here in order to comprehend its impact quantitatively. Fig. 8(c) shows the impact of $R_{\mathrm{mc}}$ on the delay at various interconnect levels. As can be observed, even for $R_{\mathrm{mc}}$ value as large as $20 \mathrm{k} \Omega$ per conducting channel, the delay penalty is 


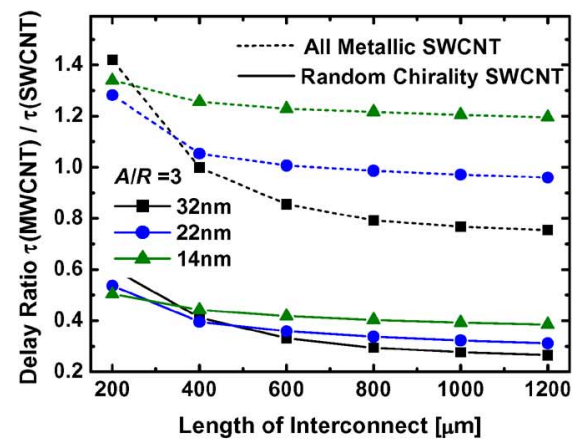

(a)

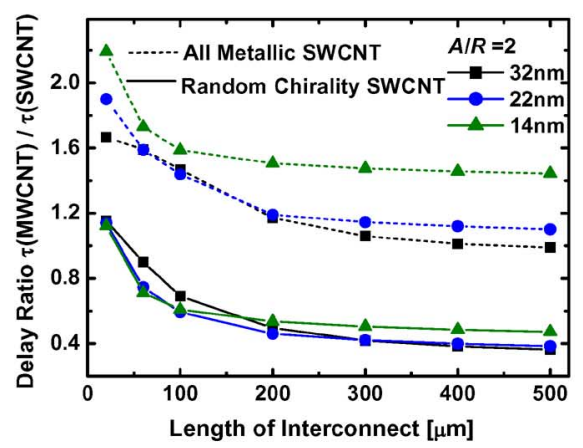

(b)

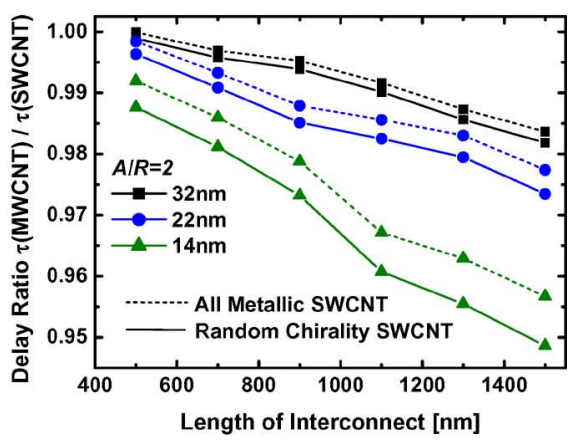

(c)

Fig. 9. Signal delay ratios of MWCNT interconnect with respect to SWCNT interconnect at (a) global, (b) intermediate, and (c) local levels for 32-, 22-, and 14-nm technology nodes, respectively. The diameters of MWCNTs correspond to the wire widths (shown in Table I). The number of MWCNTs is equal to the aspect ratio. The broken lines correspond to SWCNTs which are all metallic, and the solid lines are for SWCNTs with random chiralities.

less than $6 \%$, which indicates that $R_{\mathrm{mc}}$ does not have significant impact on MWCNT interconnects. Similar conclusion has been drawn for SWCNT bundles in the case of long interconnects [14], [15]. Moreover, the effect of $R_{\mathrm{mc}}$ is relatively larger in intermediate level interconnects since their driver resistances are smaller than those of the local wires and their scattering resistance is not as large as those of the global wires.

\section{MWCNT VERSUS SWCNT INTERCONNECTS}

As discussed in Section I, both SWCNT and MWCNT can be used as interconnect material. From the aforementioned discussions and the results from previous SWCNT interconnect modeling works [14]-[17], it can be concluded that both SWCNT and MWCNT interconnects can achieve better performance than $\mathrm{Cu}$ wires for long lengths. However, it is important to investigate how SWCNT and MWCNT compare with each other. This is due to the fact that, at present, several fabrication challenges need to be overcome for densely packed near$100 \%$ metallic SWCNT bundles. Hence, the performances of SWCNT- and MWCNT-based interconnects are compared here.

The cross-sectional view of SWCNT bundles is shown in Fig. 5(c). For densely packed bundles, the intervals between adjacent SWCNTs $(d)$ can be assumed to be $0.34 \mathrm{~nm}$, which is the van der Waals gap. The number of SWCNTs in the bundle is calculated as follows:

$$
N_{\mathrm{CNT}}=N_{W} N_{H}-\operatorname{Inter}\left[N_{H} / 2\right]
$$

where $N_{W}$ and $N_{H}$ are obtained from the equations shown in Fig. 5(c), and the operator "Inter[.]" indicates that only the integer part is to be considered. Here, the diameter of SWCNT is assumed to be $1 \mathrm{~nm}$, which has also been used in previous SWCNT works [14]-[17]. Two cases of SWCNT bundles are examined: 1) All the SWCNTs in the bundles are metallic, and 2) the SWCNTs in the bundles have random chiralities, i.e., only $1 / 3$ of the SWCNTs are metallic, whereas the rest $2 / 3$ of the SWCNT are semiconducting and do not contribute to the conductance. It is important to note that while the fraction of metallic SWCNTs in a bundle can be increased beyond $1 / 3$ by using some selective techniques (such as in [36]), achieving $100 \%$ metallic SWCNTs remains challenging. Another issue of
SWCNT bundle is the density of nanotubes. The low fraction of metallic SWCNTs in a bundle in the second case can also be regarded as low nanotube density in the bundle. Thus, not only the impact of chirality but also the impact of density can be demonstrated through the following performance analysis.

Same structure (shown in Fig. 5) and geometrical parameters (shown in Table I) used in Section III are employed for SWCNT bundles at the global, intermediate, and local interconnect levels, respectively. The resistance, inductance, and quantum capacitance of SWCNT can be calculated based on (7), (12), and $(13)$, respectively (with channel number $=2$ ). For densely packed SWCNT bundles, their electrostatic capacitance has slight difference with the capacitance of $\mathrm{Cu}$ wire [17]. Hence, it is reasonable to assume that the electrostatic capacitances of dense SWCNT bundles are equal to those of $\mathrm{Cu}$ wires. Here, similar to the aforementioned analysis for MWCNTs, the metal-nanotube contact resistance $R_{\mathrm{mc}}$ of SWCNT bundles is ignored. The simulations for SWCNT interconnects are based on rigorous distributed "W" model in HSPICE [32].

Fig. 9(a) shows the delay comparison between MWCNT and SWCNT bundle interconnects at the global level. It is found that the chiralities (or density) of SWCNTs in the bundle have significant effect on the interconnect performance. If the SWCNTs are all metallic, SWCNT interconnects can outperform MWCNT when the diameter of MWCNT is small, which will be the case for highly scaled technologies (such as in 14-nm technology node). However, if the SWCNTs have random chiralities (or is of low density), the MWCNT interconnect can achieve much better performance than that of SWCNT bundles. It is also shown that for larger dimension and longer interconnects, MWCNTs can be advantageous. This can be explained based on the smaller resistivity of large-diameter MWCNTs, as shown in Fig. 6. Similar phenomena can also be observed at the intermediate level, as shown in Fig. 9(b).

For local interconnects, the comparison between MWCNT and SWCNT interconnects is shown in Fig. 9(c). Unlike the intermediate and global interconnects, the chiralities of SWCNTs in bundles have little effect on their performance. When the chiralities of SWCNTs are changed from all metallic to random, the delay ratios change by only $<5 \%$. Note that although the resistivity of MWCNT interconnects for short lengths is much larger than that of SWCNT, it still marginally 
outperforms the SWCNTs. This can be explained based on the fact that the electrostatic capacitance of MWCNT interconnects is smaller than that of SWCNT bundles (equal to $\mathrm{Cu}$ wires, as shown in Table I). At the local level, because of large driver resistance, the difference in resistance between MWCNT and SWCNT interconnects gets screened, and the interconnect capacitance plays a more dominant role than the interconnect resistance. Furthermore, as shown in Fig. 9(c), the situation (in terms of the delay ratio) is reverse for small-diameter MWCNTs as compared to Fig. 9(a) and (b). This could be due to the fact that MWCNTs with smaller diameter have lower resistivity at shorter lengths, as shown in Fig. 6.

To summarize, in order for SWCNT bundles to be competitive (or better) with respect to MWCNT interconnects, dense SWCNT bundles with high metallic fraction are necessary.

\section{CONCLUSION}

The applicability of MWCNTs as an interconnect candidate in future design of integrated circuits has been explored theoretically in this paper. With large diameter, each shell in MWCNT has long MFP and contributes to the conductance, even if the shell is of semiconducting chirality. A comprehensive equivalent distributed circuit model has been presented, based on which the performance of MWCNT interconnects has been evaluated and compared with that of $\mathrm{Cu}$ interconnects as well as SWCNT interconnects. For long (intermediate and global levels) interconnects, MWCNT interconnects show significant improvement in signal delay as compared to $\mathrm{Cu}$ wires (as low as $15 \%$ of $\mathrm{Cu}$ delay). It is also predicted that the improvement will be enhanced with further technology scaling. For short local interconnects, the delay of MWCNT interconnects is marginally larger than that of $\mathrm{Cu}$ (by 1\%-6\%). When compared to SWCNT bundles, if SWCNTs can be densely packed and have high (near 100\%) metallic fraction, MWCNT interconnects do not exhibit any evident advantages. However, in the case of SWCNT bundles with random chiralities (or low density), MWCNT interconnects can outperform SWCNT bundles, even at long lengths. Therefore, since MWCNTs are easier to fabricate with less concern about chirality and density control, they can be attractive for immediate use as horizontal wires in VLSI, including local, intermediate, and global level interconnects.

\section{ACKNOWLEDGMENT}

Two authors, H. Li and K. Banerjee, would like to thank Dr. F. Kreupl of Qimonda AG, Munich, Germany, for useful technical feedback.

\section{REFERENCES}

[1] K. Banerjee and N. Srivastava, "Are carbon nanotubes the future of VLSI interconnections?” Proc. IEEE/ACM Design Autom. Conf., 2006, pp. 809-814.

[2] P. G. Collins, M. Hersam, M. Arnold, R. Martel, and P. Avouris, "Current saturation and electrical breakdown in multiwalled carbon nanotubes," Phys. Rev. Lett., vol. 86, no. 14, pp. 3128-3131, Apr. 2001.

[3] K. M. Liew, C. H. Wong, X. Q. He, and M. J. Tan, "Thermal stability of single and multi-walled carbon nanotubes," in Phys. Rev. B, Condens. Matter, vol. 71, Feb. 2005, p. 075424.
[4] P. L. McEuen, M. S. Fuhrer, and H. K. Park, "Single-walled carbon nanotube electronics," IEEE Trans. Nanotechnol., vol. 1, no. 1, pp. 78-85, Mar. 2002.

[5] B. Q. Wei, R. Vajtai, and P. M. Ajayan, "Reliability and current carrying capacity of carbon nanotubes," Appl. Phys. Lett., vol. 79, no. 8, pp. 1172-1174, Aug. 2001.

[6] N. Srivastava and K. Banerjee, "Interconnect challenges for nanoscale electronic circuits," TMS J. Mater, vol. 56, no. 10, pp. 30-31, Oct. 2004.

[7] N. Srivastava and K. Banerjee, "A comparative scaling analysis of metallic and carbon nanotube interconnections for nanometer scale VLSI technologies," in Proc. 21st Int. VLSI Multilevel Interconnect Conf., Sep. 2004, pp. 393-398.

[8] S. Im, N. Srivastava, K. Banerjee, and K. E. Goodson, "Scaling analysis of multilevel interconnect temperatures for high-performance ICs," IEEE Trans. Electron Device, vol. 52, no. 12, pp. 2710-2719, Dec. 2005.

[9] F. Kreupl, A. P. Graham, M. Liebau, G. S. Duesberg, R. Seidel, and E. Unger, "Carbon nanotubes for interconnect applications," in IEDM Tech. Dig., 2004, pp. 683-686.

[10] J. Li, Q. Ye, A. Cassell, H. T. Ng, R. Stevens, J. Han, and M. Meyyappan, "Bottom-up approach for carbon nanotube interconnects," Appl. Phys. Lett., vol. 82, no. 15, pp. 2491-2493, Apr. 2003.

[11] M. Nihei, D. Kondo, A. Kawabata, S. Sato, H. Shioya, M. Sakaue, T. Iwai, M. Ohfuti, and Y. Awano, "Low-resistance multi-walled carbon nanotube vias with parallel channel conduction of inner shells," in Proc. Interconnect Technol. Conf., 2005, pp. 234-236.

[12] S. Sato, M. Nihei, A. Mimura, A. Kawabata, D. Kondo, H. Shioya, T. Iwai, M. Mishma, M. Ohfuti, and Y. Awano, "Novel approach to fabricating carbon nanotube via interconnects using size-controlled catalyst nanoparticles," in Proc. Interconnect Technol. Conf., 2006, pp. 230-232.

[13] P. J. Burke, "Luttinger liquid theory as a model of the gigahertz electrical properties of carbon nanotubes," IEEE Trans. Nanotechnol., vol. 1, no. 3, pp. 129-144, Sep. 2002.

[14] N. Srivastava and K. Banerjee, "Performance analysis of carbon nanotube interconnects for VLSI applications," in Proc. IEEE/ACM Int. Conf. ICCAD, 2005, pp. 383-390.

[15] N. Srivastava, R. V. Joshi, and K. Banerjee, "Carbon nanotube interconnects: Implications for performance, power dissipation and thermal management," in IEDM Tech. Dig., 2005, pp. 257-260.

[16] A. Naeemi, R. Sarvari, and J. D. Meindl, "Performance comparison between carbon nanotube and copper interconnects for gigascale integration (GSI)," IEEE Electron Device Lett., vol. 26, no. 2, pp. 84-86, Feb. 2005.

[17] A. Naeemi and J. D. Meindl, "Design and performance modeling for single-walled carbon nanotubes as local, semiglobal, and global interconnects in gigascale integrated systems," IEEE Trans. Electron Devices, vol. 54, no. 1, pp. 26-37, Jan. 2007.

[18] P. G. Collins, M. S. Arnold, and P. Avouris, "Engineering carbon nanotubes and nanotube circuits using electrical breakdown," Science, vol. 292, no. 5517, pp. 706-709, Apr. 2001.

[19] H. J. Li, W. G. Lu, J. J. Li, X. D. Bai, and C. Z. Gu, "Multichannel ballistic transport in multiwall carbon nanotubes," Phys. Rev. Lett., vol. 95, no. 8, p. 086601, Aug. 2005.

[20] A. Naeemi and J. D. Meindl, "Compact physical models for multiwall carbon-nanotube interconnects," IEEE Electron Device Lett., vol. 27, no. 5, pp. 338-340, May 2006.

[21] D. Rossi, J. M. Cazeaux, C. Metra, and F. Lombardi, "Modeling crosstalk effects in CNT bus architectures," IEEE Trans. Nanotechnol., vol. 6, no. 2, pp. 133-145, Mar. 2007.

[22] International Technology Roadmap for Semiconductors, 2005. [Online]. Available: http://public.itrs.net/

[23] J. Jiang, J. Dong, H. T. Yang, and D. Y. Xing, "Universal expression for localization length in metallic carbon nanotubes," Phys. Rev. B, Condens. Matter, vol. 64, no. 4, p. 045 409, Jul. 2001.

[24] X. Zhou, J. Y. Park, S. Huang, J. Liu, and P. L. McEuen, "Band structure, phonon scattering, and the performance limit of single-walled carbon nanotube transistors," Phys. Rev. Lett., vol. 95, no. 14, p. 146805 , Sep. 2005.

[25] W. H. Hayt and J. A. Buck, Engineering Electromagnetics, 7th ed. New York: McGraw-Hill, 2005.

[26] P. G. Collins and P. Avouris, "Multishell conduction in multiwalled carbon nanotubes," Appl. Phys. A, Solids Surf., vol. 74, no. 3, pp. 329-332, Mar. 2002.

[27] Y. G. Yoon, P. Delaney, and S. G. Louie, "Quantum conductance of multiwall carbon nanotubes," Phys. Rev. B, Condens. Matter, vol. 66, no. 7 , p. 073 407, Aug. 2002. 
[28] B. Bourlon, C. Miko, L. Forro, D. C. Glattli, and A. Bachtold, "Determination of the intershell conductance in multiwalled carbon nanotubes," Phys. Rev. Lett., vol. 93, no. 17, p. 176 806, Oct. 2004.

[29] F. W. Grover, Inductance Calculations. New York: Dover, 2004.

[30] J. F. Xu, H. Li, W. Y. Yin, J. F. Mao, and L. W. Li, "Extraction of three-dimensional interconnects using element-by-element finite element method and preconditioned conjugate gradient technique," IEICE Trans. Electron., vol. E90C, no. 1, pp. 179-188, Jan. 2007.

[31] M. Kamon, M. J. Tsuk, and J. White, "Fasthenry: A multipole-accelerated 3-D inductance extraction program," IEEE Trans. Microw. Theory Tech., vol. 42, no. 9, pp. 1750-1758, Sep. 1994.

[32] HSPICE Manuals, Synopsis Inc., Mountain View, CA, 2003.

[33] K. Banerjee and A. Mehrotra, "A power-optimal repeater insertion methodology for global interconnects in nanometer designs," IEEE Trans. Electron Devices, vol. 49, no. 11, pp. 2001-2007, Nov. 2002

[34] M. L. Mui, K. Banerjee, and A. Mehrotra, "A global interconnect optimization scheme for nanometer scale VLSI with implications for latency, bandwidth, and power dissipation," IEEE Trans. Electron Devices, vol. 51, no. 2, pp. 195-203, Feb. 2004.

[35] X.-C. Li, J.-F. Mao, H.-F. Huang, and Y. Liu, "Global interconnect width and spacing optimization for latency, bandwidth and power dissipation," IEEE Trans. Electron Devices, vol. 52, no. 10, pp. 2272-2279, Oct. 2005.

[36] R. Krupke, F. Hennrich, H. V. Lohneysen, and M. M. Kappes, "Separation of metallic from semiconducting single-walled carbon nanotubes," Science, vol. 301, no. 5631, pp. 344-347, Jul. 2003.

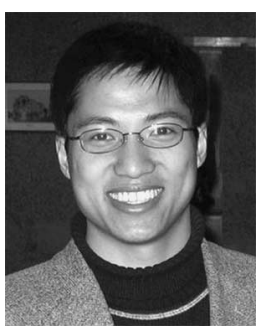

Hong Li (S'07) received the B.E. degree in electronic engineering from Nanjing University of Aeronautics and Astronautics, Nanjing, China, in 2003, and the M.S. degree in electronic engineering from Shanghai Jiao Tong University, Shanghai, China, in 2008 . He is currently working toward the Ph.D. degree in electrical and computer engineering at the University of California, Santa Barbara.

His research interests are in the design, modeling, and fabrication of carbon nanotube/nanoribbonbased VLSI interconnection technology and passive devices for high-speed mixed-signal and RF ICs.

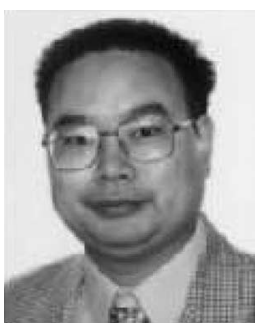

Wen-Yan Yin (M'99-SM'01) received the M.Sc. degree in electromagnetic field and microwave technique from Xidian University, Xi'an, China, in 1989, and the $\mathrm{Ph} . \mathrm{D}$. degree in electrical engineering from Xi' an Jiaotong University, Xi' an, China, in 1994.

From 1993 to 1996, he was an Associate Professor with the Department of Electronic Engineering, Northwestern Polytechnic University, Xi' an, China. From 1996 to 1998, he was a Research Fellow with the Department of Electrical Engineering, Duisburg University, Duisburg, Germany. In December 1998, he was a Research Fellow with the Monolithic Microwave Integrated Circuit Modeling and Packing Laboratory, Department of Electrical Engineering, National University of Singapore (NUS), Singapore. In March 2002, he was with Temasek Laboratories, NUS, as a Research Scientist and as the Project Leader of high-power microwave and ultrawide-band electromagnetic compatibility/electromagnetic interference (EMC/EMI). Since April 2005, he has been a Chair Professor in electromagnetic fields and microwave techniques with the School of Electronic, Information and Electrical Engineering, Shanghai Jiao Tong University (SJTU), Shanghai, China, where he is also currently the Director of the Center for Microwave and RF Technologies. $\mathrm{He}$ has authored or coauthored more than 130 international journal articles including 15 book chapters. His current research interests include electromagnetic characteristics of complex media and their applications in engineering, EMC, EMI, and electromagnetic protection for on-chip passive and active MM (RF) IC devices, ultrawide-band interconnects and signal integrity, and nanoelectronics.

Dr. Yin is the Technical Chair of the Electrical Design of Advanced Packaging and Systems-2006 (EDAPS'06), technically sponsored by the IEEE CPMT Subcommittee.

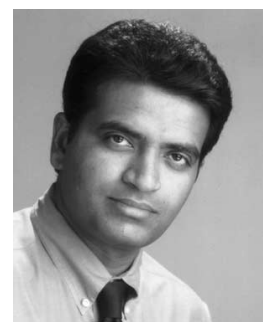

Kaustav Banerjee (S'92-M'99-SM'03) received the $\mathrm{Ph} . \mathrm{D}$. degree in electrical engineering and computer sciences from the University of California, Berkeley, in 1999.

From 1999 to 2001, he was a Research Associate with the Center for Integrated Systems, Stanford University, Stanford, CA. He has also held summer/ visiting positions with Texas Instruments Incorporated, Dallas, from 1993 to 1997, and the Swiss Federal Institute of Technology, Lausanne, Switzerland, in 2001. Since July 2002, he has been with the Faculty of the Department of Electrical and Computer Engineering, University of California, Santa Barbara (UCSB), where he is currently a Professor. At UCSB, he directs the Nanoelectronics Research Lab and is an Affiliated Faculty at the California NanoSystems Institute. From February to August 2002, he was a Visiting Faculty with the Circuits Research Lab of Intel, Hillsboro, OR. His research has been chronicled in over 150 journal and refereed international conference papers. He has also coedited a book entitled Emerging Nanoelectronics: Life with and after CMOS (New York, NY: Springer-Verlag, 2004) and two book chapters related to 3-D integrated circuits. His current research interests focus on nanometer-scale issues in highperformance/low-power VLSI as well as on circuits and systems issues in emerging nanoelectronics.

Dr. Banerjee is a member of the Nanotechnology Committee of the IEEE Electron Devices Society. He has served on the technical program committees of several leading IEEE and ACM conferences, including IEDM, DAC, ICCAD, and IRPS. He has also served on the organizing committee of ISQED, at various positions including Technical Program Chair in 2002 and General Chair in 2005. He has received a number of awards in recognition of his work, including the ACM SIGDA Outstanding New Faculty Award in 2004, a Research Award from the Electrostatic Discharge Association in 2005, a Best Paper Award at the Design Automation Conference in 2001, an Outstanding Student Paper Award at the VLSI/ULSI Multilevel Interconnection Conference in 2005, and an IEEE Micro Top Picks Award in 2006, which recognizes the top ten papers from premiere computer architecture conferences every year.

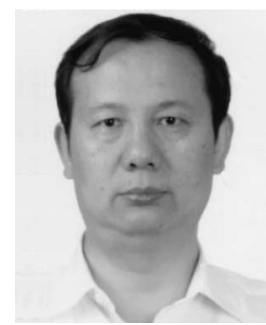

Jun-Fa Mao (M'92-SM'98) was born in 1965. He received the B.S. degree in radiation physics from the University of Science and Technology of National Defense, Changsha, China, in 1985, the M.S. degree in experimental nuclear physics form Shanghai Institute of Nuclear Research, Shanghai, China, in 1988, and the Ph.D. degree in electronic engineering from Shanghai Jiao Tong University, Shanghai, China, in 1992 .

Since 1992, he has been with the faculty of the Department of Electronic Engineering, Shanghai Jiao Tong University, Shanghai, China, where he is currently a Professor. He was a Visiting Scholar with the Chinese University of Hong Kong, Sha Tin, Hong Kong, from 1994 to 1995, and a Postdoctoral Researcher with the University of California, Berkeley, from 1995 to 1996. He was a Topic Expert of the High-Tech Program of China during 2001-2003 and an Associate Dean of the School of Electronic, Information and Electrical Engineering, Shanghai Jiao Tong University, from 1999 to 2005. He has authored or coauthored more than 200 papers and coauthored one book. His current research interests include the interconnect problem of high-speed integrated circuits and novel microwave components and circuits.

Dr. Mao is a Cheung Kong Scholar of the Ministry of Education, China, an Associate Director of the Microwave Society of China Institute of Electronics, and the 2007-2008 Chair of the IEEE Shanghai Section. He received the Second-Class National Natural Science Award of China in 2004 and the FirstClass Natural Science Award of Shanghai in 2005. 